\title{
Modeling and Analysis of a Piezoelectric Energy Harvester with Varying Cross-Sectional Area
}

\author{
Maiara Rosa and Carlos De Marqui Junior \\ Department of Aeronautical Engineering, Engineering School of São Carlos, University of São Paulo, \\ Avenida Trabalhador São-Carlense, 400 Pq Arnold Schimidt, 13566-590 São Carlos, SP, Brazil \\ Correspondence should be addressed to Carlos De Marqui Junior; demarqui@sc.usp.br
}

Received 12 July 2013; Accepted 3 March 2014; Published 9 September 2014

Academic Editor: Miguel M. Neves

Copyright (C) 2014 M. Rosa and C. De Marqui Junior. This is an open access article distributed under the Creative Commons Attribution License, which permits unrestricted use, distribution, and reproduction in any medium, provided the original work is properly cited.

\begin{abstract}
This paper reports on the modeling and on the experimental verification of electromechanically coupled beams with varying crosssectional area for piezoelectric energy harvesting. The governing equations are formulated using the Rayleigh-Ritz method and Euler-Bernoulli assumptions. A load resistance is considered in the electrical domain for the estimate of the electric power output of each geometric configuration. The model is first verified against the analytical results for a rectangular bimorph with tip mass reported in the literature. The experimental verification of the model is also reported for a tapered bimorph cantilever with tip mass. The effects of varying cross-sectional area and tip mass on the electromechanical behavior of piezoelectric energy harvesters are also discussed. An issue related to the estimation of the optimal load resistance (that gives the maximum power output) on beam shape optimization problems is also discussed.
\end{abstract}

\section{Introduction}

The interest in converting vibrations into usable electrical energy has increased over the past years [1-5]. Vibration based energy harvesting is particularly useful for wireless sensor nodes and remotely operated systems with limited energy source. The aim is to provide electrical energy for such systems by using the vibrations available in their environment. Although different transduction mechanisms can be used to convert vibrations into electricity, the recent literature shows that piezoelectric transduction has drawn the most attention $[1,3,5]$.

The literature on piezoelectric energy harvesting includes different models to represent the behavior of electromechanically coupled harvesters. Such models range from lumped parameter models [6, 7] to Rayleigh-Ritz type approximate distributed parameter models [7-9] as well as analytical distributed parameter solution attempts $[10,11]$. The analytical distributed parameter solutions for unimorph [12] and bimorph [13] piezoelectric energy harvester configurations with closed-form expressions have been presented. The convergence of the Rayleigh-Ritz type electromechanical solution $[7,9]$ to the analytical solution given by Erturk and Inman [12] was reported by Elvin and Elvin [14] when a sufficient number of admissible functions were used.

The investigation into alternative configurations of electromechanical beams has also been reported in the literature. Erturk et al. [15] presented a linear distributed parameter model for predicting the electromechanical behavior of an L-shaped piezoelectric energy harvester configuration. A broadband harvester can be obtained when the first two natural frequencies of the L-shaped beam are properly tuned. The use of tapered cantilevers in order to improve the electromechanical behavior of piezoelectric energy harvesters has also been investigated [16-22]. The shape is changed from the basic rectangular configuration towards a tapered or reversed tapered geometry and the main motivation is to increase the electrical power output. The modeling of electromechanically coupled beams with nonuniform width is presented in Dietl and Garcia [21]. An optimal beam shape is determined by 
an optimization code. In the cited paper [21], as well as in $[2,16,22]$, the expression $1 / \omega C_{p}$ (where $\omega$ is the excitation frequency and $C_{p}$ is the equivalent capacitance of piezoceramic layers) is employed for the optimum load resistance (which gives maximum power output) of a piezoelectric energy harvester. In general, the authors approximate the eigenvalues and eigenvector of electromechanically coupled beams with nonuniform width to the ones corresponding to rectangular shape beams [18-20]. Recently, the solution of eigenvalue problem of nonuniform width beams by using the differential quadrature method has been presented and the effects of beam shape on the structural natural frequencies and mode shapes are discussed [23]. An important aspect, the effect of load resistance on the electroelastic behavior of variable-shaped harvesters, is only considered in Ayed et al. [23].

An issue related to the estimate of the optimum load from the equation $1 / \omega C_{p}$ was previously discussed [24] for a rectangular (or squared) energy harvester configuration. The piezoceramic layer of a piezoelectric energy harvester can be represented as a current source in parallel with its internal capacitance (Norton representation) or as a voltage source in series with its internal capacitance (Thévenin representation). For instance, in Norton representation, $R_{\mathrm{opt}}=1 / \omega C_{p}$ is obtained only if a constant current amplitude oscillating at a frequency $\omega$ is assumed. However, regarding the electromechanical behavior of a piezoelectric energy harvesting previously discussed in the literature $[13,24,25]$, the current source in Norton representation is not constant, but it depends on the load resistance, since the vibration response also depends on the load resistance. The expression $R_{\mathrm{opt}}=1 / \omega C_{p}$ could be obtained from the coupled equations that govern a piezoelectric energy harvester [24] only if the electromechanical coupling term was artificially removed from the mechanical equation.

This paper reports on the modeling and experimental verification of electromechanically coupled beams with varying cross-sectional area (tapered or reversed tapered) for energy harvesting. The governing equations are formulated by the Rayleigh-Ritz method and Euler-Bernoulli assumptions. A load resistance is considered in the electrical domain of the problem for the estimate of the electric power output. The model is first verified against analytical electromechanically-coupled results of a rectangular bimorph cantilever with tip mass under base excitation reported in the literature [13]. The frequency response functions (FRFs) obtained using the model presented in this work are compared with the FRFs obtained analytically in [13]. The model is also experimentally verified for a tapered bimorph cantilever with tip mass. Finally, the effects of a varying cross-sectional area (changing from a rectangular beam to a tapered or reversed tapered configuration) and tip mass on the electrical power output of piezoelectric energy harvesters are discussed. The effects of the incorrect estimate of the optimal load resistance (which provides the maximum power output) from the expression $R_{\mathrm{opt}}=1 / \omega C_{p}$ on both the electromechanical behavior of each geometric configuration and the beam shape optimization problem are discussed.

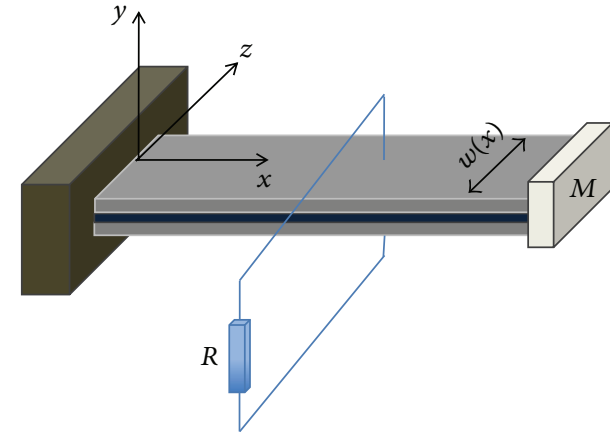

FIGURE 1: A bimorph piezoelectric energy harvester under clampedfree boundary conditions.

\section{Mathematical Model of a Piezoelectric Energy Harvester with Varying Cross- Sectional Area}

The derivation provided in this section is for a bimorph piezoelectric beam of uniform width along its length (rectangular) or nonuniform width along its length (tapered or reversed tapered). The bimorph harvester has a brass substructure bracketed by two piezoceramic layers as shown in Figure 1 (for the rectangular beam). Each piezoelectric layer is covered by continuous conductive electrodes that can be connected either in series (when piezoceramic layers are poled in the opposite direction) or in parallel (when piezoceramic layers are poled in the same direction). In this work, the pairs of electrodes covering each piezoceramic layer are connected in series. In Figure 1, $R$ is the resistive load, $w(x)$ is the width of the beam along the length $(x)$, and $M$ is the tip mass attached to the free end of the harvester.

The combination of Hamilton's principle [26] and the Rayleigh-Ritz method based on the Euler-Bernoulli beam assumptions is used in the modeling approach of this paper. The generalized Hamilton's principle was applied by Hagood et al. [27], who combined the Rayleigh-Ritz method with the Euler-Bernoulli beam theory for active structural control. The Rayleigh-Ritz formulation used by Hagood et al. [27] was also implemented by duToit et al. [7] and Sodano et al. [8] for predicting the electric power output of electromechanically coupled Euler-Bernoulli beams in energy harvesting problems. Dietl and Garcia [21] combined the RayleighRitz method with the Euler-Bernoulli beam theory to model electromechanically coupled beams with a varying crosssectional area in energy harvesting problems.

In the Euler-Bernoulli beam theory, the motion is restricted to the transverse direction and the only nonzero component of the displacement field $\mathbf{u}$ is $y(x, t)$. Furthermore, the beam strain is given by $y(x, t)$ and its partial derivatives. In the Rayleigh-Ritz procedure, the displacement $y(x, t)$ of the beam can be written as the summation of the modes and the temporal coordinate of the displacement as

$$
y(x, t)=\boldsymbol{\varphi}(x)^{T} \mathbf{q}(t),
$$

where $\varphi(x)$ is a matrix of assumed mode shapes and $\mathbf{q}(t)$ is the temporal coordinate of displacement. Here, the mode 
shapes are assumed to be an acceptable solution to an EulerBernoulli beam under a clamped-free condition,

$$
\widetilde{\boldsymbol{\varphi}}_{k}(x)=\cos \beta_{k} \frac{x}{L}-\cosh \beta_{k} \frac{x}{L}-\sigma_{k}\left(\sin \beta_{k} \frac{x}{L}-\sinh \beta_{k} \frac{x}{L}\right),
$$

where $L$ is the length of the beam and $\sigma_{k}$ is expressed as

$$
\sigma_{k}=\frac{\sin \beta_{k}-\sinh \beta_{k}+\beta_{k} r\left(\cos \beta_{k}-\cosh \beta_{k}\right)}{\cos \beta_{k}+\cosh \beta_{k}-\beta_{k} r\left(\sin \beta_{k}-\sinh \beta_{k}\right)},
$$

where $\beta_{k}$ is the natural frequency of the $k$ th mode obtained from the equation given by

$$
\frac{1}{r}(\cos \beta \cosh \beta+1)+\beta(\cos \beta \sinh \beta-\sin \beta \cosh \beta)=0,
$$

where $r$ is the ratio of the tip mass to the mass of the beam (substructure and piezoceramic layers).

The general form for the $k$ th mode shape must satisfy the following equation

$$
\int_{0}^{L} \varphi_{k}^{2}(x) \rho_{l} w(x) d x+M \varphi_{k}^{2}(L)=1,
$$

where the mode shape is obtained as

$$
\varphi_{k}(x)=\frac{\widetilde{\varphi}_{k}(x)}{\sqrt{\int_{0}^{L} \widetilde{\varphi}_{k}^{2}(x) d x+r \widetilde{\varphi}_{k}^{2}(L)}},
$$

and $\rho_{l}$ is the equivalent mass density given by

$$
\rho_{l}=\rho_{s} h_{s}+2 \rho_{p} h_{p}
$$

where $\rho$ is the mass density, $h$ is the thickness of the layer and the subscripts $s$ and $p$ represent, respectively, the substructure and the piezoceramic layers.

Since piezoceramic layers are poled in the thickness direction, the nonzero electric field component $(E)$, which is assumed to be uniform along the thickness direction, is expressed as

$$
E=-\frac{\partial \psi}{\partial z}=-\frac{V}{2 h_{p}}
$$

where the electric potential $(\psi)$ is assumed to vary linearly across the electrodes and $V$ is the voltage across the electrodes.

The previous definitions for mechanical and electrical variables should be used to define the terms in Hamilton's principle (please check Dietl and Garcia [21] or De Marqui Jr. et al. [25] for details) to provide the electromechanically coupled equations governing the beam:

$$
\begin{gathered}
\mathbf{M} \ddot{\mathbf{q}}(t)+\mathbf{C} \dot{\mathbf{q}}(t)+\mathbf{K q}(t)-\boldsymbol{\Theta} V(t)=\mathbf{F} \\
C_{p} \dot{V}(t)+\frac{V(t)}{R}+\boldsymbol{\Theta}^{T} \dot{\mathbf{q}}(t)=0
\end{gathered}
$$

where $\mathbf{M}$ is the mass matrix, $\mathbf{C}$ is the damping matrix, $\mathbf{K}$ is the stiffness matrix, $\boldsymbol{\Theta}$ is the electromechanical coupling matrix, $\mathbf{F}$ is the vector of mechanical forces (where $\mathbf{F}=\mathbf{p}^{*} a(t)$, where $a(t)$ is the base acceleration in function of time and $\mathbf{p}^{*}$ is the input matrix to be defined later), $T$ represents the matrix transpose when superscripted, an overdot represents the time derivative, $R$ is the load resistance, $\mathbf{q}$ is the vector of modal mechanical displacements, $V(t)$ is the voltage in function of the time, and $C_{p}$ is the effective capacitance.

The mass matrix is defined as

$$
\mathbf{M}=\int_{0}^{L} \rho_{l} w(x) \boldsymbol{\varphi}(x) \boldsymbol{\varphi}^{T}(x) d x+M \boldsymbol{\varphi}(L) \boldsymbol{\varphi}^{T}(L)
$$

and the stiffness matrix is defined as

$$
\mathbf{K}=\frac{I_{0 w}}{s_{p}^{E}}\left[\int_{0}^{L} w(x) \boldsymbol{\varphi}^{\prime \prime}(x) \boldsymbol{\varphi}^{\prime \prime T}(x) d x\right],
$$

where $s_{p}^{E}$ is the compliance measured in a constant electric field, $(")$ represents the space derivative, and $I_{0 w}$ is given by

$$
I_{0 w}=\frac{I_{z z}(x)}{w(x)},
$$

where $I_{z z}(x)$ is the moment of inertia.

The damping matrix is assumed to be proportional to the mass and stiffness matrices:

$$
\mathbf{C}=\alpha \mathbf{M}+\beta \mathbf{K},
$$

where $\alpha$ and $\beta$ are the constants of proportionality.

The capacitance for a bimorph harvester in series connection case is given by

$$
C_{p}=\frac{\varepsilon_{33}^{S}}{2 h_{p}} \int_{0}^{L} w(x) d x
$$

where $\varepsilon_{33}^{S}$ is the dielectric constant evaluated at constant strain for an Euler-Bernoulli beam as

$$
\varepsilon_{33}^{S}=1730 \varepsilon_{0}-\frac{d_{31}}{E_{p}}
$$

and $\varepsilon_{0}$ is the permittivity in free space and $d_{31}$ is the piezoelectric coupling coefficient and $E_{p}$ is Young's modulus of the piezoceramic.

The electromechanical coupling matrix is given as

$$
\Theta=-\left(h_{s} h_{p}+h_{p}^{2}\right) \frac{d_{31}}{2 s_{p}^{E} h_{p}}\left[\int_{0}^{L} w(x) \varphi^{\prime \prime}(x) d x\right]
$$

and the input matrix is

$$
\mathbf{p}^{*}=\int_{0}^{L} \rho_{l} w(x) \boldsymbol{\varphi}(x) d x+M \boldsymbol{\varphi}(L),
$$

with all variables previously defined.

Expressions for the electromechanical FRFs (voltage across the resistive load, current passing through the resistive 
TABLE 1: Geometric and material properties of the bimorph harvester.

\begin{tabular}{|c|c|c|c|}
\hline Length of the beam [mm] & 50.8 & Mass density of the substructure $\left[\mathrm{kg} / \mathrm{m}^{3}\right]$ & 9000 \\
\hline Width of the beam on the clamped end [mm] & 31.8 & Mass density of the PZT $\left[\mathrm{kg} / \mathrm{m}^{3}\right]$ & 7800 \\
\hline Width of the beam on the free end [mm] & 31.8 & Tip mass $[\mathrm{kg}]$ & 0.012 \\
\hline Thickness of the substructure [mm] & 0.14 (each) & Proportional constant $\alpha(\mathrm{rad} / \mathrm{s})$ & 14.65 \\
\hline Thickness of the PZT [mm] & 0.26 (each) & Proportional constant $\beta$ (s/rad) & $10^{-5}$ \\
\hline Young's modulus of the substructure [GPa] & 105 & Piezoelectric coupling coefficient $d_{31}[\mathrm{pm} / \mathrm{V}]$ & -190 \\
\hline Young's modulus of the PZT-5A [GPa] & 66 & Permittivity of free space $[\mathrm{pF} / \mathrm{m}]$ & 8.854 \\
\hline
\end{tabular}

load, electrical power output, and relative tip motion) can be obtained from the equations of motion ((9) and (10)). The excitation is due to the harmonic motion of the base in the transverse direction, $w_{B}=Y_{0} e^{j \omega t}$ (where $w_{B}(t)$ is the base displacement, $Y_{0}$ is its amplitude, $\omega$ is the excitation frequency, and $j$ is the unit imaginary number), and the voltage output-to-base acceleration FRF can be obtained as

$$
\begin{aligned}
& \frac{V(t)}{a(t)} \\
& =\frac{V(t)}{-\omega^{2} Y_{0} e^{j \omega t}}=j \omega\left(\frac{1}{R}+j \omega C_{p}\right)^{-1} \boldsymbol{\Theta}^{T} \\
& \quad \times\left[-\omega^{2} \mathbf{M}+j \omega \mathbf{C}+\mathbf{K}+j \omega\left(\frac{1}{R}+j \omega C_{p}\right)^{-1} \boldsymbol{\Theta} \Theta^{T}\right]^{-1} \mathbf{p}^{*}
\end{aligned}
$$

and the electric current FRF is obtained by dividing the voltage FRF by the load resistance of the electrical circuit and the electrical peak power FRF (since the voltage FRF is the peak voltage FRF) is the product of the voltage and current FRFs.

The relative tip motion FRF is defined as the ratio of the amplitude of the displacement at the tip of the beam (relative to the base) to the amplitude of the base displacement input and it is obtained from (9) and (10) as

$$
\begin{aligned}
& \frac{w_{\text {rel }}}{Y_{0} e^{j \omega t}} \\
& \quad=\omega^{2}\left[-\omega^{2} \mathbf{M}+j \omega \mathbf{C}+\mathbf{K}+j \omega\left(\frac{1}{R}+j \omega C_{p}\right)^{-1} \Theta \Theta^{T}\right]^{-1} \mathbf{p}^{*}
\end{aligned}
$$

and the tip velocity FRF is defined as the ratio of the amplitude of velocity at the tip of the beam (relative to the fixed frame) to the gravitational acceleration. This FRF is easily obtained from the expression of the relative tip motion FRF by using

$$
v_{\text {rel }}=-\frac{j g}{\omega}\left(1+\frac{w_{\text {rel }}(L, t)}{Y_{0}}\right),
$$

where $g$ is the gravitational acceleration.

\section{Case Studies}

This section presents three case studies and the electromechanically coupled model described in Section 2 is employed.
In the first case, the model is verified against the analytical results of a bimorph cantilever with tip mass reported in the literature [13]. The experimental verification of the model is then reported for a tapered bimorph cantilever with tip mass. Finally, a discussion regarding the calculation of the optimal load resistance (for maximum power output) is presented. The effects of varying cross-sectional area, tip mass, and estimate of optimal load resistance on the electromechanical behavior and shape optimization problems of piezoelectric energy harvesters are also discussed. It is important to mention that, in the following discussions, the power output is normalized per base acceleration (in terms of gravitational acceleration), which is assumed to be smaller than that which would cause failure in the different piezoelectric energy harvesters considered in this work.

3.1. Verification against the Analytical and Experimental Results for a Rectangular Bimorph Configuration. In the first case study, the results obtained from our electromechanical model presented for a rectangular cantilevered bimorph with a tip mass under base excitation are compared with the single mode analytical predictions of the closed-form solution presented by Erturk and Inman [13]. The bimorph harvester configuration has a brass substructure bracketed by two PZT-5A layers. The piezoceramic layers are poled in the opposite directions and therefore the combination of the layers to the electrical load results in the series connection case. The numerical input data of the bimorph is shown in Table 1.

The voltage FRF is defined here as the voltage output per gravitational acceleration $\left(g=9.81 \mathrm{~m} / \mathrm{s}^{2}\right)$ to be in agreement with the analytical voltage FRFs given by Erturk and Inman [13]. Equation (19) is easily modified to provide voltage output per $g$. The voltage FRFs for the first mode of the harvester obtained from our model are plotted in Figure 2(a) along with the analytical solution and experimental results for eight different values of load resistance $(1,6.7,11.8,22,33,47,100$, and $470 \mathrm{k} \Omega$ ). The voltage output increases with increasing load resistance for all excitation frequencies according to the present model and the analytical predictions. The analytical model as well as our model has predicted such frequencies as $45.7 \mathrm{~Hz}$ and $48.2 \mathrm{~Hz}$, respectively.

The mechanical vibration FRFs of the bimorph piezoelectric harvester obtained by the present model and the analytical model are shown in Figure 2(b). The tip velocity FRF ((20) and (21)) is defined as the ratio of the amplitude of velocity at the tip of the beam (relative to the fixed 


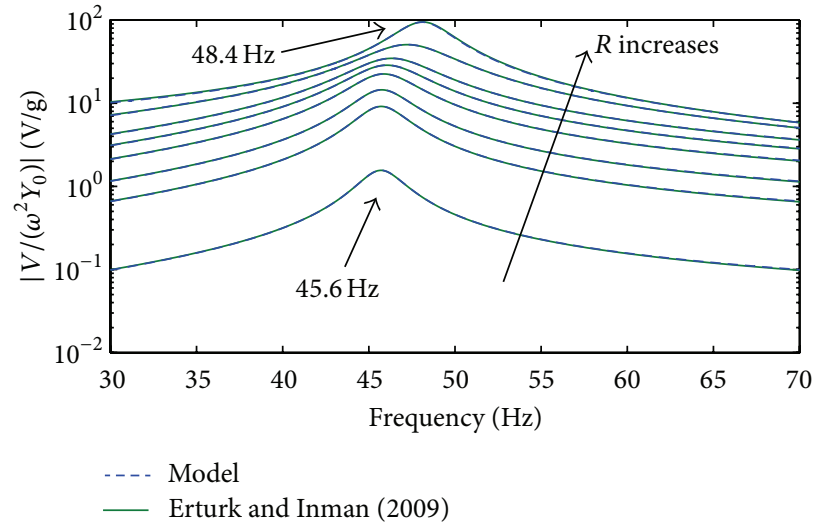

(a)

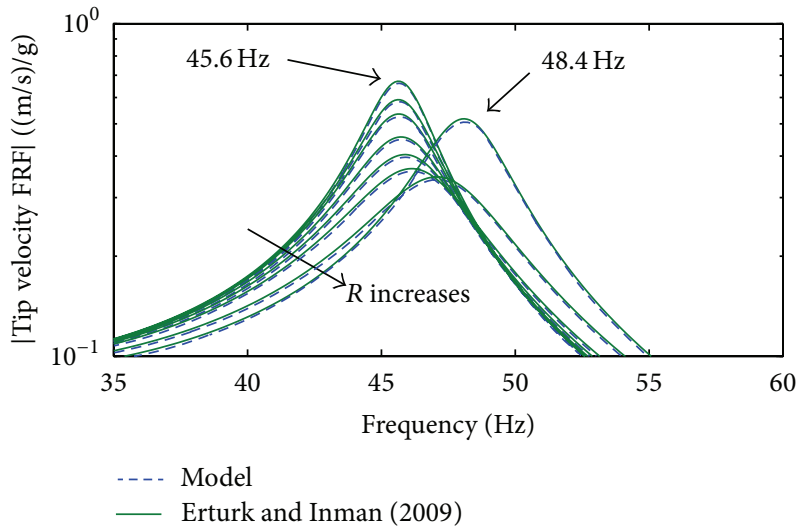

(b)

FigURE 2: Voltage FRF (a) and tip velocity (FRF) (b) for eight values of load resistance.

TABLE 2: Geometric and material properties of the tapered bimorph harvester.

\begin{tabular}{lclc}
\hline Length of the beam $[\mathrm{mm}]$ & 50.8 & Mass density of the substructure $\left[\mathrm{kg} / \mathrm{m}^{3}\right]$ & 9000 \\
Width of the beam at the clamped end $[\mathrm{mm}]$ & 31.5 & Mass density of the PZT $\left[\mathrm{kg} / \mathrm{m}^{3}\right]$ & 7800 \\
Width of the beam at the free end $[\mathrm{mm}]$ & 7.25 & Tip mass $[\mathrm{kg}]$ & 0.00164 \\
Thickness of the substructure $[\mathrm{mm}]$ & $0.14($ each $)$ & Proportional constant $\alpha(\mathrm{rad} / \mathrm{s})$ & 8.79 \\
Thickness of the PZT $[\mathrm{mm}]$ & $0.26($ each $)$ & Proportional constant $\beta(\mathrm{s} / \mathrm{rad})$ & $6.10^{-6}$ \\
Young's modulus of the substructure $[\mathrm{GPa}]$ & 105 & Piezoelectric coupling coefficient $d_{31}[\mathrm{pm} / \mathrm{V}]$ & -190 \\
Young's modulus of the PZT-5A [GPa & 66 & Permittivity of free space $[\mathrm{pF} / \mathrm{m}]$ & 8.854 \\
\hline
\end{tabular}

frame) to the gravitational acceleration. The mechanical FRFs obtained by the present model are in agreement with the analytical results. The vibration amplitude at the short circuit resonance frequency is attenuated as the load resistance is increased up to $100 \mathrm{k} \Omega$ due to the resistive shunt damping effect associated with power generation. Approximately after the value of $100 \mathrm{k} \Omega$, increasing load resistance amplifies the vibration amplitude at the open circuit resonance frequency.

\subsection{Verification against the Experimental Results for a Tapered} Bimorph with Tip Mass. In this second case study, the results from our approximate model for a tapered piezoelectric energy harvester are verified against experimental results. The bimorph harvester configuration has a brass substructure bracketed by two PZT-5A layers. The piezoceramic layers are poled in the opposite directions and therefore the series connection case is studied. The geometric and material properties for the tapered beam are given in Table 2. The width of the beam at the clamped end is larger than the width at the free end and it is assumed to vary linearly along the length of the harvester.

Small magnets were attached at the free end of the tapered harvester as a tip mass in the experiments. The base acceleration was measured at the clamped end (Accelerometer Model 352C22, PCB Piezotronics), which is connected to a shaker (Model 4810, Brüel \& Kjær). A digital laser vibrometer (Model PDV-100, Polytec) measures the tip velocity at the free end. The electromechanical behavior is investigated by using three different resistive loads $(1 \mathrm{k} \Omega, 50 \mathrm{k} \Omega$, and $1 \mathrm{M} \Omega$ ) and the electromechanical FRFs were acquired through a Data Acquisition System (Photon II All in One System, LDS Dactron). Figure 3 shows the experimental setup.

The voltage FRFs for the first mode of the tapered harvester obtained from our model and the experimental results are plotted in Figure 4(a). The experimental short circuit and open circuit resonance frequencies for the tapered harvester are $179.1 \mathrm{~Hz}$ and $189.1 \mathrm{~Hz}$, respectively. The present model has predicted such frequencies as $178.8 \mathrm{~Hz}$ and $188.9 \mathrm{~Hz}$, respectively. The tip velocity FRFs obtained by the present model are in agreement with those of the experimental results, as shown in Figure 4(b).

3.3. Effects of Tip Mass, Beam Shape, and Load Resistance on the Electromechanical Behavior of a Piezoelectric Energy Harvester. In the third case study, the effects of a varying cross-sectional area (changing from a rectangular beam to a tapered or reversed tapered configuration) and tip mass on the electrical power output of piezoelectric energy harvesters are discussed. Issues related to the estimate of the optimal load resistance by using the expression $R_{\mathrm{opt}}=1 / \omega C_{p}$ on the power output of each geometric configuration and on beam shape optimization problems are also discussed. The piezoceramic layers of the bimorph are poled in opposite directions and therefore the series connection case is investigated. The numerical input data of the base case studied in this section (rectangular beam) are given in Table 1.

Two different conditions are investigated in this case. In the first, the width of the clamped end $(x=0)$ is constant 


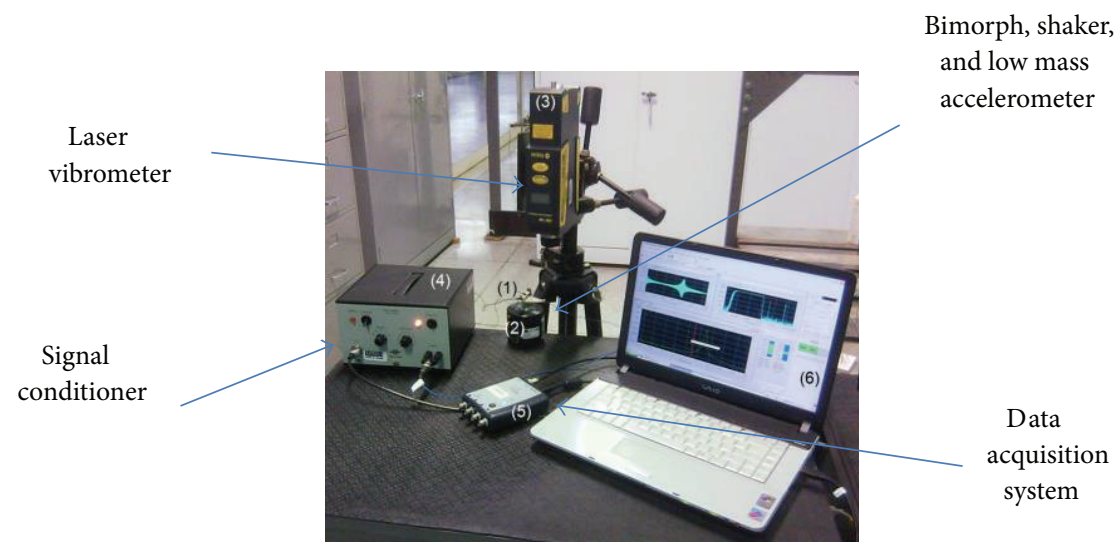

FIGURE 3: Experimental setup used for the verification of relations for a tapered beam.

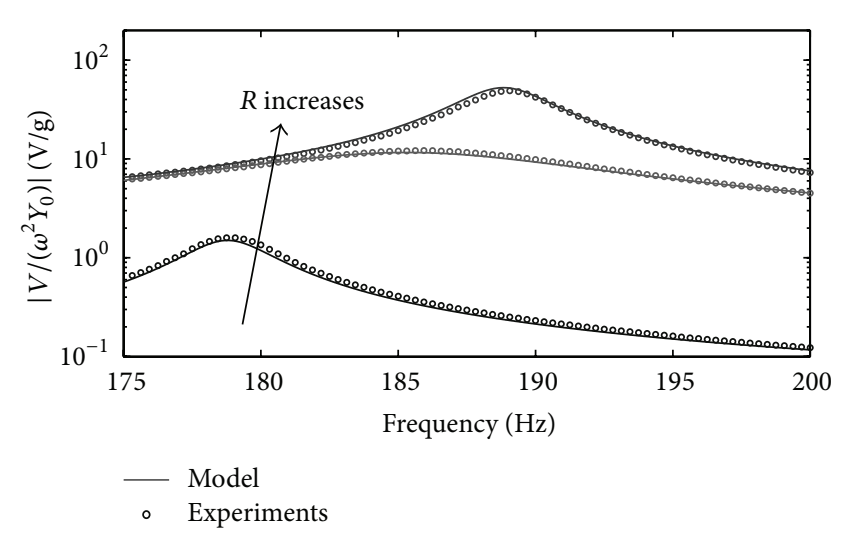

(a)

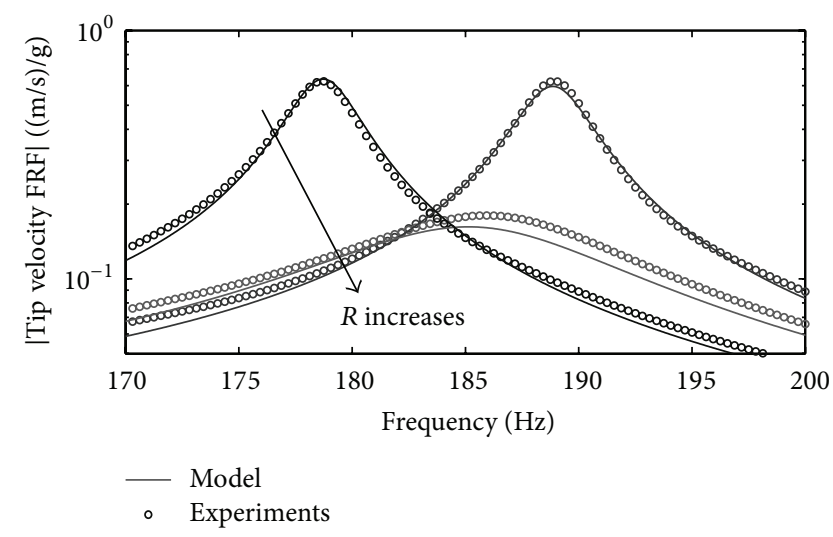

(b)

FIGURE 4: Model and experimental voltage FRFs (a) and tip velocity FRFs (b) for three values of load resistance.

$\left(w_{0}=w(0)=31.8 \mathrm{~mm}\right)$ and the width at the tip can be modified (tapered beam). The width (or cross-section) is linearly modified along the span, from $w_{0}$ at the clamped end to $w(L)=P w_{0}$ at the free end. $P$ may assume values from 0 (triangular beam) to 1 (rectangular shape), as shown in Figure 5(a). In the second case, the width of the free end $(x=L)$ is constant $\left(w(L)=w_{0}=31.8 \mathrm{~mm}\right)$ and the width of the clamped end can be modified (reversed tapered beam). The width (or cross-section) is linearly modified along the span from $w(L)=w_{0}$ at the clamped end to $w(0)=Q w_{0}$ at the free end, where $Q$ may assume values from 0 (reversed tapered beam) to 1 (rectangular shape), as shown in Figure 5(b). The thickness and length of the composite section (substructure and piezoceramic layers) are constant in both cases and a tip mass is assumed in the free end.

The power output in each case (tapered or reversed tapered) is obtained for a range of values of tip mass and $P$ or $Q$ (for each case), as well as for the optimum load resistance of the short circuit resonance frequency of each configuration. Although small values of parameters $P$ and $Q$ are assumed in the analyses, note that $P \rightarrow 0$ and
$Q \rightarrow 0$ can lead to practical issues related to fabrication and testing (tip mass position or clampling). The optimum load resistances are estimated considering (1) the Norton or Thévenin representation of the piezoelectric layers and (2) an expression for the optimum load obtained from (9) and (10) [23]. As previously discussed, when the Norton or Thévenin representations of the piezoelectric layers are assumed, the optimum load resistance is

$$
R_{\mathrm{opt}}=\frac{1}{\omega_{k} C_{p}},
$$

where $\omega_{k}$ is the short circuit resonance frequency of the desired mode. Such an expression is obtained when the backward coupling is neglected in the equations that govern the piezoelectric energy harvester. Therefore, the shift from short to open circuit resonance frequency as well as the shunt damping effect (trends reported in the previous case studies) are not observed when the load resistance is changed from short to open circuit conditions [23]. 


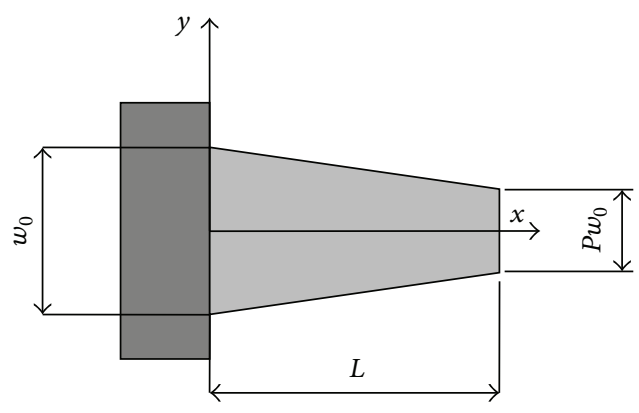

(a)

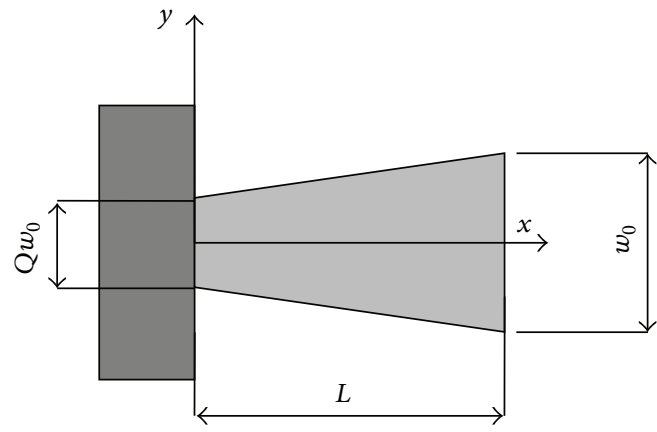

(b)

FIGURE 5: Standard shape modification of a bimorph piezoelectric harvester by parameters $P$ and $Q$.

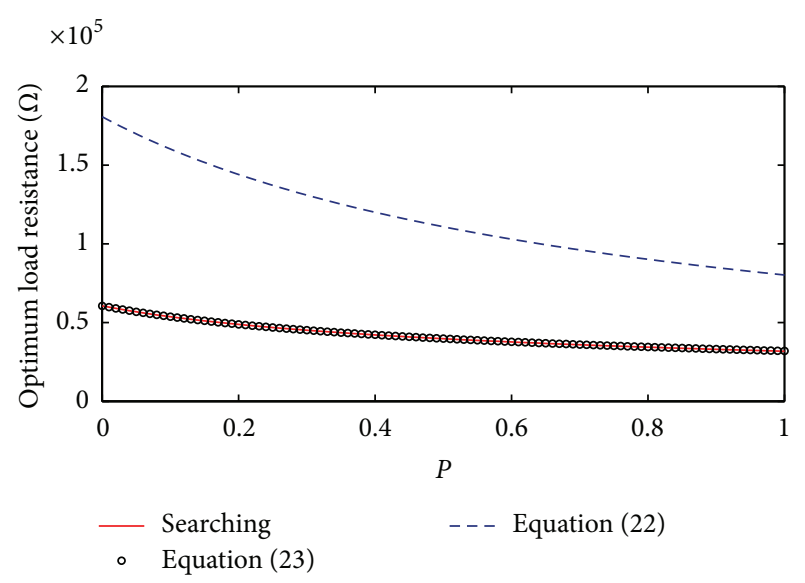

FIgURE 6: Variation of optimum load resistance with parameter $P$.

By considering the electromechanically coupled equations ((9) and (10)) one should obtain the expression for the optimum load as [23]

$$
R_{\mathrm{opt}}=\frac{1}{\omega_{k} C_{p}\left[1+\left(\gamma_{k} / 2 \zeta_{k}\right)^{2}\right]} \quad \text { where } \gamma_{k}=\frac{\Theta_{k}}{C_{p} \omega_{k}}
$$

and $\omega_{k}$ is the short circuit resonance frequency of the desired mode and $\zeta_{k}$ is the damping ratio of the same mode and $\Theta_{k}$ is the modal electromechanical coupling of the mode. The optimum load resistance can also be searched by the calculation of the power output for a wide range of load resistance until the optimal one is reached $[15,24]$ when the system is excited at a target frequency. For instance, Figure 6 shows the variation of the optimum load resistance with parameter $P$ for a piezoelectric energy harvester with tip mass (basic data given in Table 1) excited at the short circuit resonance frequency. The same load resistance is obtained by searching for the optimum one and by using (23). However, inaccurate predictions are obtained from (22).

The variation of power output (per squared based acceleration) with parameter $P$ and tip mass is shown in Figure 7. The excitation is due to the harmonic motion of the clamped end in the transverse direction at the short circuit resonant frequency of the first vibration mode of each configuration. The maximum power output displayed in Figure 7(a) is obtained from the power FRF defined in Section 2 (obtained from the harmonic assumption in (9) and (10)) and the optimum load resistance (for each configuration) is calculated by using (23). Power increases with increasing tip mass for any geometric configuration (from rectangular to triangular harvester or $1 \leq P \leq 0$ ). This is the expected behavior, since the fundamental vibration mode of the harvester is considered in this base excitation problem. It is important to note that the forcing term in the base excitation is related to the inertia of the body itself; therefore, larger mass values result in larger strains and power output. Figure 7(a) shows that power output increases with increasing $P$ for any tip mass. Therefore, the maximum power output is obtained from the rectangular piezoelectric energy harvester. This result contradicts the conclusions of other papers [16-22]; however, the effect of load resistance on the electromechanical behavior of a piezoelectric energy harvester is an important aspect that has not been considered previously. Ayed et al. [23] report the tapered beam as the optimum one. However, no experimental verification is provided and the open circuit condition $\left(R=10^{6} \Omega\right)$ is reported as the optimum load resistance.

The maximum power output displayed in Figure $7(\mathrm{~b})$ is obtained from the power FRF defined from (9) and (10) with the electrical term in the mechanical domain (9) artificially set to zero. Therefore, the optimum load resistance (of each configuration) is calculated by (22). It is noteworthy that the power output in Figure 7(b) is larger than that in Figure 7(a). It is important to remember that the shunt damping effect was neglected in the simulations of Figure 7(b) (since electric feedback was neglected in (9)). The maximum power output in Figure $7(b)$ is obtained for the largest tip mass and for $P=0.23$. The simple representation of the piezoelectric layers as constant electrical sources (Norton or Thévenin representations) leads to the incorrect estimate of the optimum load resistance (Figure 6) as well as to the incorrect optimum shape that provides the maximum power output in a beam optimization problem.

Finally, the variation of the power output (per squared based acceleration) with parameter $Q$ and tip mass is shown 


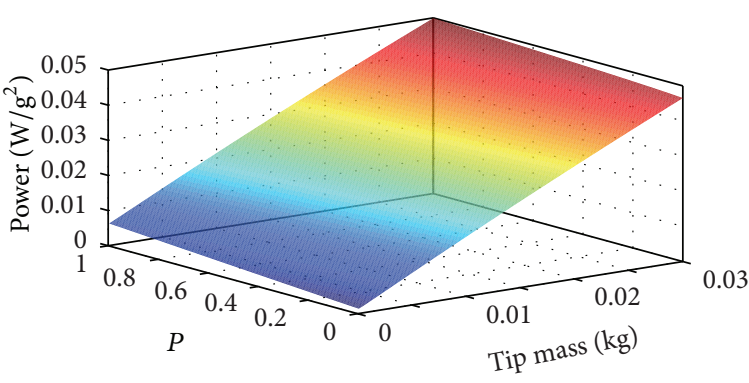

(a)

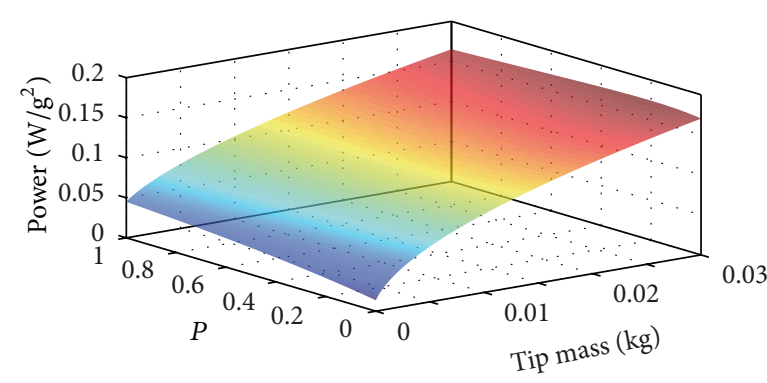

(b)

FIGURE 7: Variation of power output (per squared based acceleration) with parameter $P$ and tip mass.

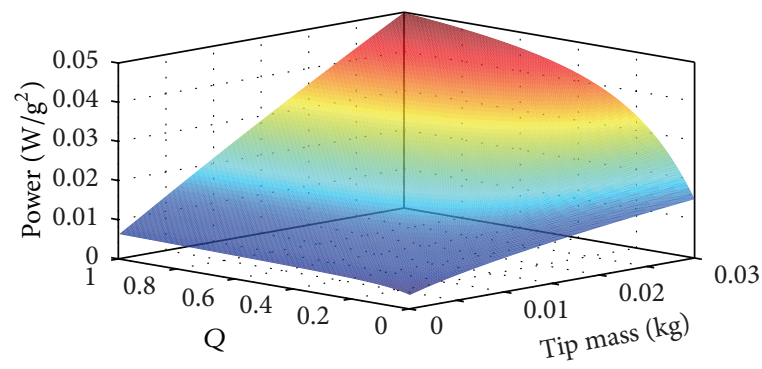

(a)

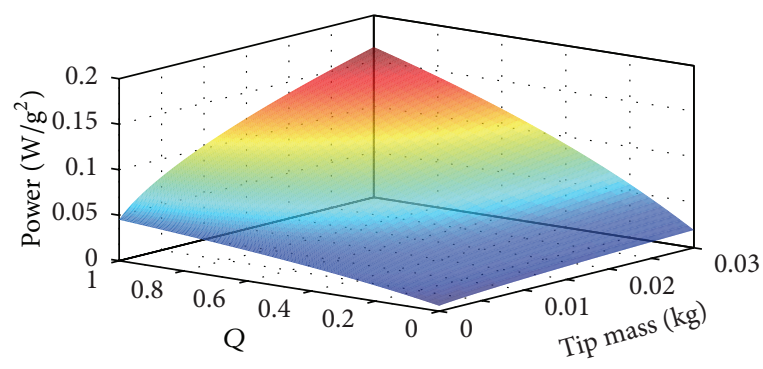

(b)

FIGURE 8: Variation of power output (per squared based acceleration) with parameter $Q$ and tip mass.

in Figure 8. The system is excited at the short circuit resonant frequency of the first vibration mode of each configuration. The maximum power output displayed in Figure 8(a) is obtained by using the power FRF expression defined in Section 2 (obtained from the harmonic assumption in (9) and (10)) and the optimum load resistance (for each configuration) calculated by (23). Power increases with increasing tip mass for any geometric configuration (from rectangular to reversed tapered beam or $1 \leq Q \leq 0$ ). Power output increases with increasing $Q$ for any tip mass considered in the simulations. Therefore, the maximum power output is obtained from the rectangular piezoelectric energy harvester. One should also note that power output dramatically drops (for any tip mass) with decreasing $Q$. In such a case, piezoelectric material is being removed from the region of maximum strain of a cantilever, which reduces the electromechanical coupling. The maximum power output displayed in Figure 8(b) is obtained from the power FRF defined from (9) and (10) with the electrical term in the mechanical domain (9) artificially set to zero. The optimum load resistance (for each configuration) is calculated with (22). The power output in Figure 8(b) is larger than that in Figure 8(a). The shunt damping effect is neglected in the simulations of Figure 8(b) (since electric feedback is neglected in (9)). The power output is quite similar in Figures 8(a) and 8(b) when $Q \rightarrow 0$. In this region, the electromechanical coupling decreases with decreasing $Q$ and the effect of the electrical feedback in (9) is negligible. Therefore, for systems with small electromechanical coupling $(\boldsymbol{\theta})$, the optimum load resistances obtained from
(22) and (23) are similar (or identical when $\boldsymbol{\theta} \rightarrow 0$ in (23)) and the effect on the power output is negligible.

\section{Conclusions}

The modeling and experimental verification of electromechanically coupled beams with uniform and varying crosssectional areas have been reported for energy harvesting. The combination of Hamilton's principle and the Rayleigh-Ritz method based on the Euler-Bernoulli beam assumptions is used in the modeling approach. The electromechanical model was first verified against the analytical and experimental results for a rectangular bimorph under base excitation reported in the literature. The electromechanical vibration and voltage FRFs obtained from the presented model are in a very good agreement with those obtained from the analytical solution and experiments. In the second case, the model was successfully verified against the experimental results of a tapered bimorph with tip mass.

The effects of a varying cross-sectional area and tip mass on the electromechanical behavior of piezoelectric energy harvesters were also discussed for two conditions-tapered and reversed tapered beams. Issues related to the determination of the optimum load resistance and the consequences on beam shape optimization problems have also been addressed. When the electric term is neglected in the mechanical equation, the resulting expression for the optimum load resistance (for maximum power) is inaccurate, especially for 
systems with large electromechanical coupling. Moreover, the power output is overestimated, since the shunt damping effect is not present, and an incorrect optimum shape that gives the maximum power output is obtained in a beam shape optimization problem.

\section{Conflict of Interests}

The authors declare that there is no conflict of interests regarding the publication of this paper.

\section{Acknowledgments}

The authors gratefully acknowledge CNPQ and FAPEMIG for partially funding the present research work through INCT-EIE and the support of the Sao Paulo Research Foundation (FAPESP) (2011/11711-0).

\section{References}

[1] H. A. Sodano, D. J. Inman, and G. Park, "A review of power harvesting from vibration using piezoelectric materials," Shock and Vibration Digest, vol. 36, no. 3, pp. 197-205, 2004.

[2] S. P. Beeby, M. J. Tudor, and N. M. White, "Energy harvesting vibration sources for microsystems applications," Measurement Science and Technology, vol. 17, no. 12, pp. R175-R195, 2006.

[3] S. Priya, "Advances in energy harvesting using low profile piezoelectric transducers," Journal of Electroceramics, vol. 19, no. 1, pp. 165-182, 2007.

[4] S. R. Anton and H. A. Sodano, "A review of power harvesting using piezoelectric materials (2003-2006)," Smart Materials and Structures, vol. 16, no. 3, pp. R1-R21, 2007.

[5] K. A. Cook-Chennault, N. Thambi, and A. M. Sastry, "Powering MEMS portable devices-a review of non-regenerative and regenerative power supply systems with special emphasis on piezoelectric energy harvesting systems," Smart Materials and Structures, vol. 17, no. 4, Article ID 043001, 2008.

[6] S. Roundy, P. K. Wright, and J. Rabaey, "A study of low level vibrations as a power source for wireless sensor nodes," Computer Communications, vol. 26, no. 11, pp. 1131-1144, 2003.

[7] N. E. duToit, B. L. Wardle, and S. G. Kim, "Design considerations for MEMS-scale piezoelectric mechanical vibration energy harvesters," Integrated Ferroelectrics, vol. 71, pp. 121-160, 2005.

[8] H. A. Sodano, G. Park, and D. J. Inman, "Estimation of electric charge output for piezoelectric energy harvesting," Strain, vol. 40, no. 2, pp. 49-58, 2004.

[9] N. E. duToit and B. L. Wardle, "Experimental verification of models for microfabricated piezoelectric vibration energy harvesters," AIAA Journal, vol. 45, no. 5, pp. 1126-1137, 2007.

[10] S.-N. Chen, G.-J. Wang, and M.-C. Chien, "Analytical modeling of piezoelectric vibration-induced micro power generator," Mechatronics, vol. 16, no. 7, pp. 379-387, 2006.

[11] J. H. Lin, X. M. Wu, T. L. Ren, and L. T. Liu, "Modeling and simulation of piezoelectric MEMS energy harvesting device," Integrated Ferroelectrics, vol. 95, no. 1, pp. 128-141, 2007.

[12] A. Erturk and D. J. Inman, "A distributed parameter electromechanical model for cantilevered piezoelectric energy harvesters," Journal of Vibration and Acoustics, Transactions of the ASME, vol. 130, no. 4, Article ID 041002, 2008.
[13] A. Erturk and D. J. Inman, "An experimentally validated bimorph cantilever model for piezoelectric energy harvesting from base excitations," Smart Materials and Structures, vol. 18, Article ID 025009, 2009.

[14] N. G. Elvin and A. A. Elvin, "A general equivalent circuit model for piezoelectric generators," Journal of Intelligent Material Systems and Structures, vol. 20, no. 1, pp. 3-9, 2009.

[15] A. Erturk, J. M. Renno, and D. J. Inman, "Modeling of piezoelectric energy harvesting from an L-shaped beam-mass structure with an application to UAVs," Journal of Intelligent Material Systems and Structures, vol. 20, no. 5, pp. 529-544, 2009.

[16] S. P. Matova, M. Renaud, M. Jambunathan, M. Goedbloed, and R. Van Schaijk, "Effect of length/width ratio of tapered beams on the performance of piezoelectric energy harvesters," Smart Materials and Structures, vol. 22, no. 7, Article ID 075015, 2013.

[17] S. Roundy, E. S. Leland, J. Baker et al., "Improving power output for vibration-based energy scavengers," IEEE Pervasive Computing, vol. 4, no. 1, pp. 28-36, 2005.

[18] F. Goldschmidtboeing and P. Woias, "Characterization of different beam shapes for piezoelectric energy harvesting," Journal of Micromechanics and Microengineering, vol. 18, no. 10, Article ID 104013, 2008.

[19] L. Mateu and F. Moll, "Optimum piezoelectric bending beam structures for energy harvesting using shoe inserts," Journal of Intelligent Material Systems and Structures, vol. 16, no. 10, pp. 835-845, 2005.

[20] D. Benasciutti, L. Moro, S. Zelenika, and E. Brusa, "Vibration energy scavenging via piezoelectric bimorphs of optimized shapes," Microsystem Technologies, vol. 16, no. 5, pp. 657-668, 2010.

[21] J. M. Dietl and E. Garcia, "Beam shape optimization for power harvesting," Journal of Intelligent Material Systems and Structures, vol. 21, no. 6, pp. 633-646, 2010.

[22] F. Lu, H. P. Lee, and S. P. Lim, "Modeling and analysis of micro piezoelectric power generators for micro-electromechanicalsystems applications," Smart Materials and Structures, vol. 13, no. 1, pp. 57-63, 2004.

[23] S. B. Ayed, A. Abdelkefi, F. Najar, and M. R. Hajj, "Design and performance of variable-shaped piezoelectric energy harvesters," Journal of Intelligent Material Systems and Structures, 2014.

[24] A. Erturk, Electromechanical modeling of piezoelectric energy harvesters [Ph.D. dissertation], Department of Engineering Science and Mechanics, Virginia Polytechnic Institute and State University, , Blacksburg, Va, USA, 2009.

[25] C. De Marqui Junior Jr., A. Erturk, and D. J. Inman, "An electromechanical finite element model for piezoelectric energy harvester plates," Journal of Sound and Vibration, vol. 327, no. 12, pp. 9-25, 2009.

[26] S. H. Crandall, D. C. Karnopp, E. F. Kurtz Jr., and D. C. Pridmore-Brown, Dynamics of Mechanical and Electromechanical Systems, McGraw-Hill, New York, NY, USA, 1968.

[27] N. W. Hagood, W. H. Chung, and A. von Flotow, "Modeling of piezoelectric actuator dynamics for active structural control," Journal of Intelligent Material Systems and Structures, vol. 1, pp. 327-354, 1990. 

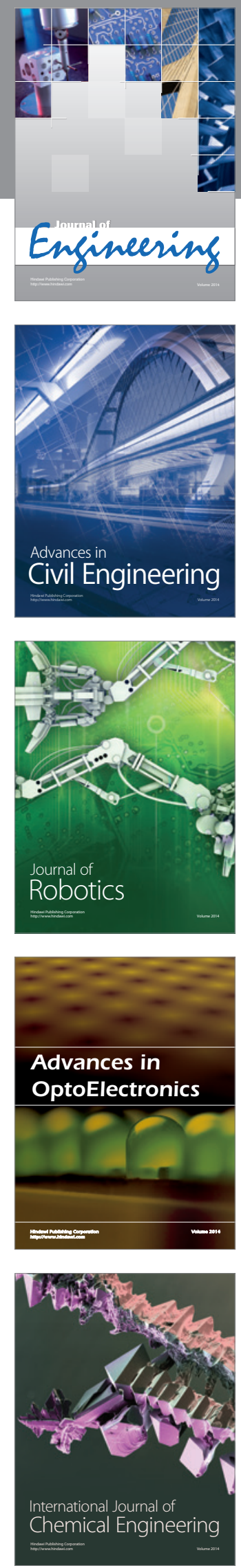

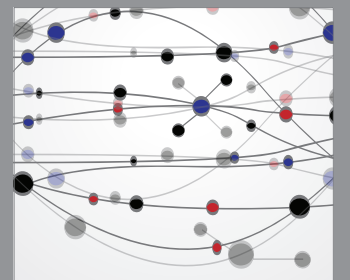

The Scientific World Journal
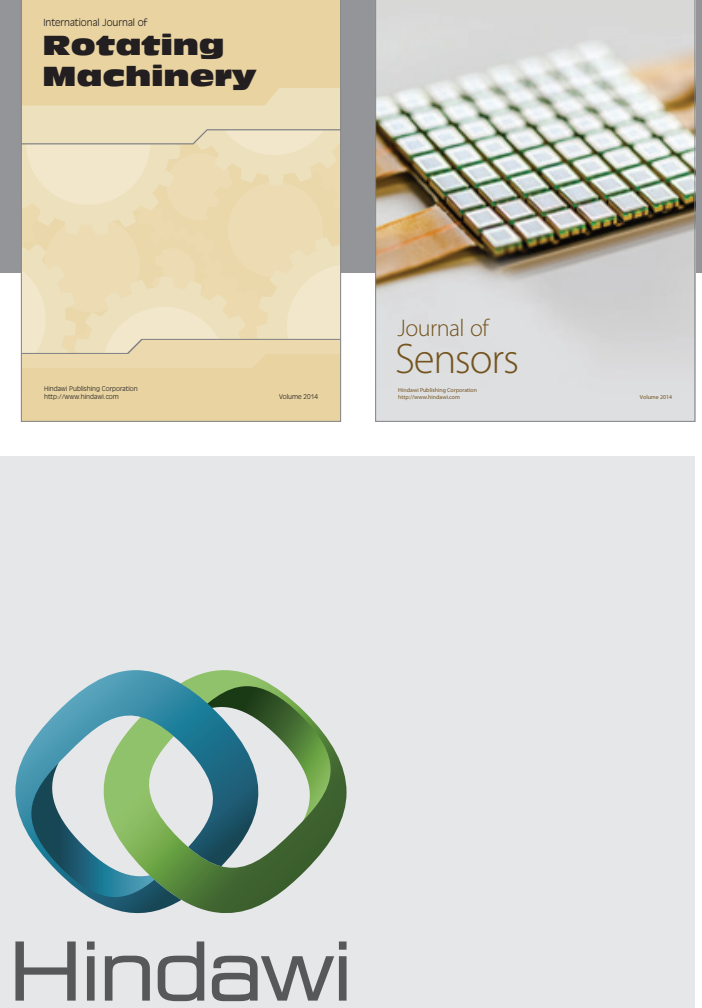

Submit your manuscripts at http://www.hindawi.com
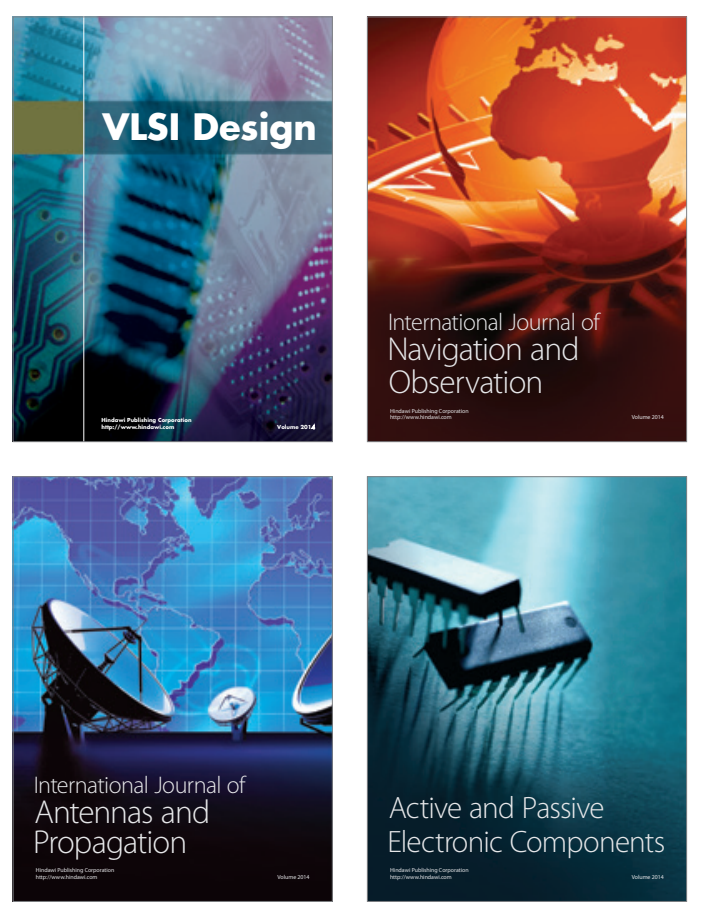
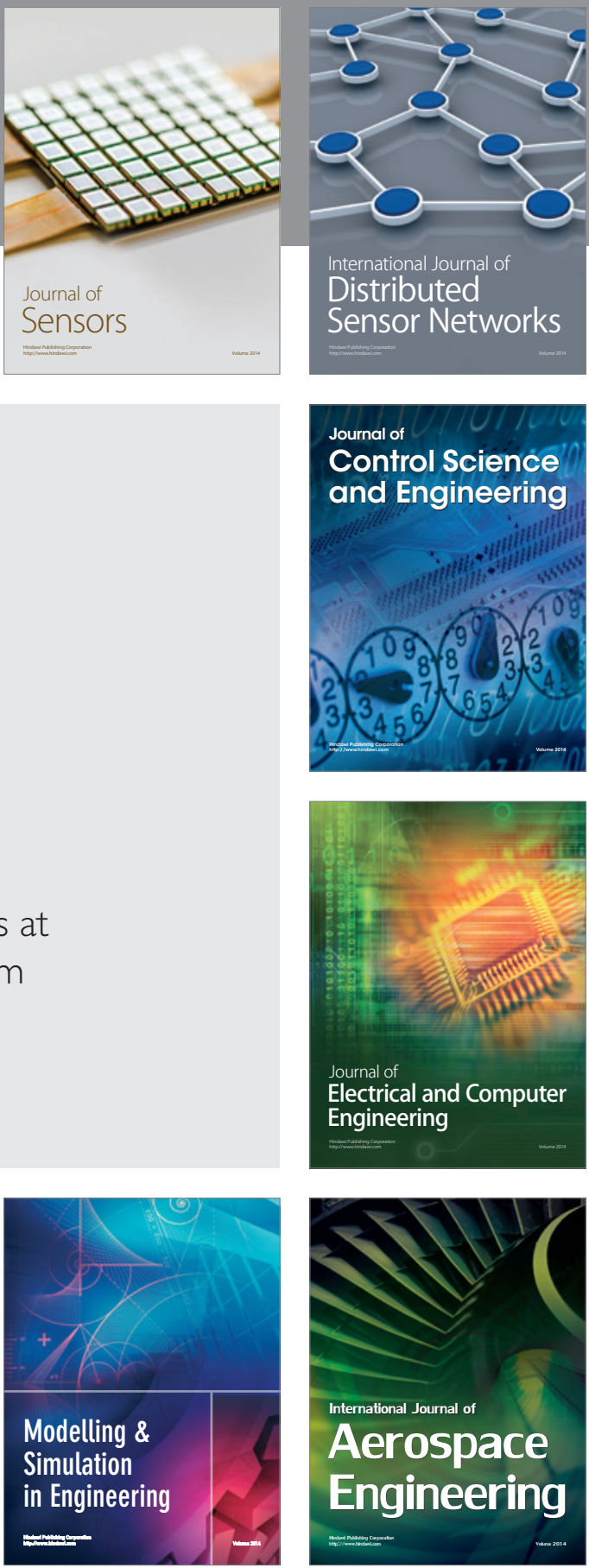

Journal of

Control Science

and Engineering
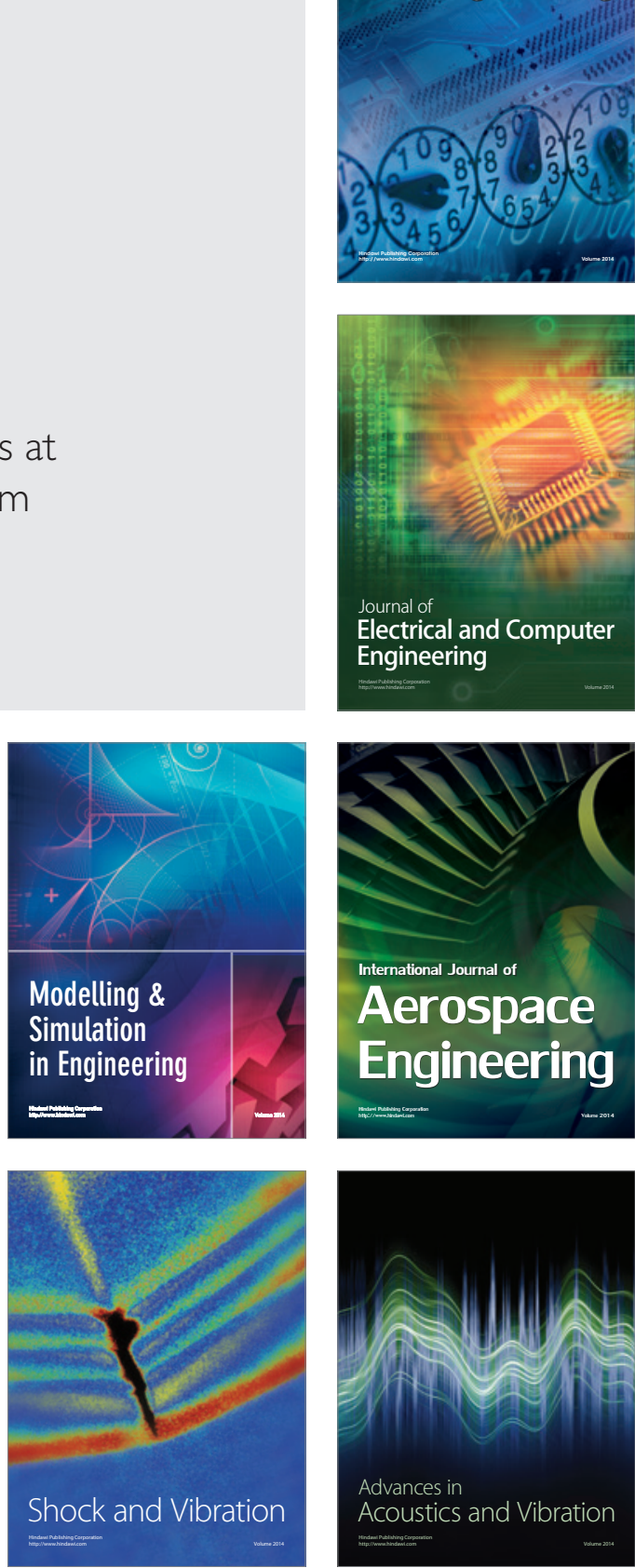